\title{
Articulación entre la matemática y el campo de acción de la Ingeniería de Diseño de Producto. Aportes de la modelación matemática
}

\author{
Articulation between mathematics and the scope of Product Design Engineering. \\ Contributions of mathematical modelling
}

\section{Alexander Castrillón-Yepes*}

(iD) https://orcid.org/0000-0002-4055-9613

Doi: 10.17533/udea.unipluri.18.2.08

Cómo citar este artículo:

Castrillón-Yepes, A. (2018). Reseña: Articulación entre la matemática y el campo de acción de la Ingeniería de Diseño de Producto. Aportes de la modelación matemática. Uni-pluriversidad, 18(2), 120-126. https://doi.org/10.17533/udea.unipluri.18.2.08 .

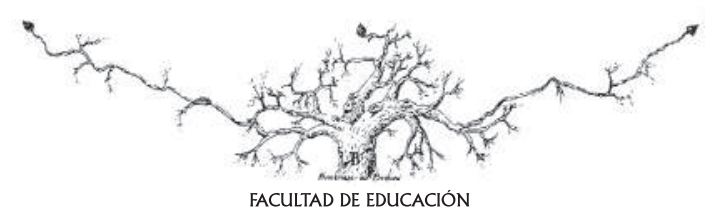

Recibido: 2018-10-07 • Aprobado: 2019-03-24

* Estudiante de la Licenciatura en Matemáticas y Física de la Universidad de Antioquia. Coordinador de la Red de Semilleros de Investigación de la Universidad de Antioquia (RedSIN UdeA). Integrante del Grupo de Investigación MATHEMA-Formación e Investigación en Educación Matemática. Correo: alexander.castrillony@udea.edu.co 


\section{Reseña de la Tesis Doctoral:}

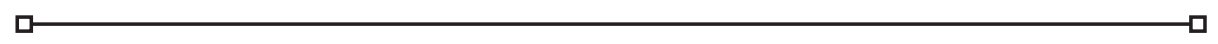

Rendón-Mesa (2016). Articulación entre la matemática y el campo de acción de la ingeniería de diseño de producto: aportes de la modelación matemática. Doctorado en Educación, Facultad de Educación, Medellín: Universidad de Antioquia.

La tesis reporta los resultados de una investigación que se desarrolló en el curso modelación matemática adscrito al programa de Ingeniería de Diseño de Producto de la Universidad EAFIT, Medellín, Colombia. La autora identificó que en este curso se hacía necesario incorporar estrategias que posibilitaran dotar de sentido la matemática en el proceso del diseño de producto. Así, bajo una perspectiva situada, la autora se ocupó de promover ambientes de modelación que posibiliten articulaciones entre la matemática y el campo de acción de la ingeniería de diseño de producto. En el estudio se configuraron dos ambientes de modelación en los cuales se reconocen los aportes del trabajo a la discusión frente a la formación ingenieril y sobre los diferentes aspectos que se deben considerar para posibilitar la articulación entre la matemática y otros campos de acción a través de la modelación.

Para definir el problema, la autora argumenta que dentro de los propósitos del curso se encuentra que los estudiantes dinamicen los conocimientos tanto matemáticos como ingenieriles, reconociendo las condiciones matemáticas que definen la creación de un diseño; sin embargo, estas condiciones no eran reconocidas por ellos y la incorporación que se realizaba de estos conceptos sobre la ingeniería no tenían una fuerte relación con el proceso de creación de una idea de diseño, sino que más bien posibilitaban el uso de modelos predeterminados para atender a las tareas propuestas (Rendón-Mesa, 2016, p. 26). Investigadores como Bravo-Bohórquez, Castañeda-Rodriguez, Hernández-Yomayusa y Hernández-Hernández (2015) han planteado que es importante generar experiencias ingenieriles a partir de la Matemática en Contexto para establecer relaciones entre el conocimiento matemático, la cotidianidad, el desempeño profesional y las ciencias; sin embargo, lo que se quiere frente al trabajo de Rendón-Mesa (2016) es llamar la atención sobre la manera en que se estructuró las nuevas versiones del curso con el fin de atender a las necesidades de formación de los estudiantes en coherencia con su campo de acción.

Bajo este contexto, la matemática en la ingeniería de diseño de producto se entendió como el medio a partir del cual el futuro ingeniero puede apropiarse de los fundamentos teóricos y prácticos que definen el diseño de un producto con la intención de atender a las necesidades de su campo de acción y a los requerimientos de los usuarios. Bajo este contexto la investigación respondió a la pregunta ¿Cómo un ambiente de modelación matemática puede promover una articulación entre la matemática y el campo de acción de la ingeniería de diseño de producto? Para ello, el trabajo se enmarcó en una perspectiva situada, pues en ella se posibilita dotar de significado el aprendizaje de los estudiantes en el campo de acción ingenieril y su futuro desempeño profesional en relación con el diseño de producto; es decir, implica vincular contextos cercanos de la ingeniería de diseño a la actividad de modelado, aun- 
que no se agota en ello, pues lo situado en la modelación matemática debe considerar que las acciones realizadas estén en correspondencia con la experiencias de los estudiantes y su cultura. No obstante, esta inmersión es compleja si se considera que para definir el diseño de productos interviene el proceso de modelación, el proceso ingenieril y el de diseño de producto.

Uno de los aportes que realiza la autora es plantear un proceso para el campo de acción a partir del reconocimiento de las componentes de los procesos de modelación, ingenieril y de diseño. En este proceso la autora considera seis subprocesos, a saber: la identificación de oportunidad que requiere de una solución, la exploración de condiciones para iniciar un plan de acción sobre esa oportunidad, la definición inicial de las condiciones para solucionar el problema, el conocimiento de la situación a partir de exploraciones teóricas o prácticas, la evaluación de condiciones o soluciones propuestas y la definición de acciones a seguir en correspondencia a las soluciones que hasta el momento se identificaron.

Este proceso planteado por la autora bajo una perspectiva situada requiere de una dialéctica entre la cultura y el sujeto, de manera que, a partir de ella, se demarquen acciones específicas (como la toma de decisiones, la generación de ideas y la construcción de argumentos) sobre una situación o fenómeno propia del diseño de productos. En este caso, se esperaba que las acciones estuvieran mediadas (y justificadas) a partir de la matemática y las acciones que impliquen el diseño de un producto; sin embargo, también se deben considerar los requerimientos formales y estructurales del producto.

En coherencia con lo anterior, se reconoce en la literatura internacional diversas características de la modelación matemática (Blomhøj, 2004; Blum, 2011; Villa-Ochoa, Castrillón-Yepes y Sánchez-Cardona, 2017) como su carácter articulador entre diferentes áreas del conocimiento y el establecimiento de modelos para predecir, analizar e interpretar fenómenos o situaciones. En ese sentido, la autora plantea que el diseño de un ambiente de modelación debe, entre otras cosas, proporcionar un contexto (el de diseño), reconocer el carácter activo del estudiante frente a su aprendizaje y desarrollar el conocimiento en acción. De esta manera, se espera que los estudiantes desarrollen habilidades que contribuyan a su futuro desarrollo profesional a través de la modelación, que incorpore procesos técnicos y teóricos, que estimulen la creatividad y resuelvan problemas. En este proceso se esperaba que se generaran apropiaciones conceptuales entre la matemática, la ingeniería y el diseño de productos.

Por otro lado, se ha promovido la incorporación de la enseñanza de la modelación matemática como estrategia curricular de manera que aporte a la motivación y comprensión de los estudiantes (Plaza, 2016); sin embargo, esto no es suficiente para atender a las necesidades de formación de los mismos, pues más allá de ser un elemento motivacional y una estrategia para la comprensión de conceptos matemáticos, la modelación situada en el contexto ingenieril (aunque no exclusivo a este), debería contribuir al establecimiento de relaciones entre esos conceptos matemáticos con los contextos propios del campo de acción, de manera que no se establezcan relaciones de subordinación entre la situación real y la matemática (Villa-Ochoa y Berrío, 2015). Este llamado a la no subordinación es realizado por Villa-Ochoa y Berrio (2015) con el fin de resaltar el papel de la modelación en los procesos de producción 
de conocimiento no matemático que no suelen ser visibles cuando la modelación solo se centra en el aprendizaje de contenidos matemáticos. La autora plantea que "una necesidad sentida en la formación de los ingenieros se encuentra en correspondencia con el uso de la matemática pero, de tal manera que tenga sentido para el futuro campo de acción" (p. 35) Bajo esta premisa, en la investigación se plantean diferentes tareas de modelación y un proyecto de modelación matemática-y-diseño que implica el establecimiento de relaciones matemáticas en contextos cercanos a la ingeniería de diseño de producto.

Las tareas propuestas por la autora además de ser cercanas al estudiante están en correspondencia con las acciones que demanda su perfil profesional. Algunas de ellas no incorporan una solución predeterminada ni inmediata; sino que, por el contrario, requieren procesos de indagación para ser resueltas, así que paralelamente a su solución se abordaban los elementos teóricos necesarios y en ocasiones el trabajo se terminó posterior a las clases. Por su parte, los proyectos realizados se pueden entender, bajo la comprensión planteada por Villa-Ochoa, Castrillón-Yepes y Sánchez-Cardona (2017), como modelación a través de proyectos. En este tipo de tareas los estudiantes seleccionaron una temática de su interés, generaron discusiones, atendieron a los requerimientos de diseño, recrearon acciones propias de un ingeniero de diseño de producto e incorporaron el uso de diferentes medios como los simuladores para apoyar argumentos y validar sus modelos.

En coherencia con lo anterior, la autora optó por la fenomenología hermenéutica para orientar la investigación, pues según ella "La intención de dicho enfoque radica en cuestionar, interpretar y llegar a la comprensión de las experiencias que ocurren.
Dicha comprensión se logra a partir del análisis reflexivo" (Rendón-Mesa, 2016, p. 99). Este análisis se centró en las maneras en que los estudiantes logran generar articulaciones entre la matemática y el campo de acción, y sobre los significados que los estudiantes dieron a las tareas (de modelación) propuestas.

El análisis en la resolución de dichas tareas permitió determinar cuatro componentes de los ámbitos de la modelación matemática en este contexto ingenieril que son necesarios para la consolidación de un ambiente de modelación. A continuación, se describe cada uno de ellos:

- Contextualización: Tiene que ver con la comprensión de los estudiantes sobre las situaciones reales en relación con su campo de acción y con el análisis del rol de la matemática en ese escenario.

- Problematización de los contextos cercanos al diseño de producto: Este espacio posibilita la reflexión sobre acciones en el proceso de modelado, se discuten las producciones, se solicitan argumentos, etc.

- Interacciones entre los estudiantes y los expertos en diversas temáticas: Aquí se focaliza con más especificidad las consideraciones que deben ser atendidas por los estudiantes para responder a una idea de diseño con apoyo de los expertos.

- Diálogos entre disciplinas: Se requiere de la integración de diferentes disciplinas, el trabajo interdisciplinar y el uso de diferentes modelos para resolver algunos problemas en la ingeniería de diseño de producto. 
Estos procesos no deben verse como componentes aislados, pues todos ellos constituyen un sistema sobre la modelación matemática en el contexto del diseño de producto y contienen elementos complejos que se relacionan con otros; por ejemplo, se deben considerar los requerimientos formales y funcionales del diseño de un producto (estos incluyen practicidad, ergonomía, seguridad, durabilidad, entre otros) en cada uno de las componentes, las matemáticas necesarias para la producción de modelos, aunque sean provisionales, los aportes de otras áreas para el diseño del producto y el sentido del modelo a la luz de los requerimientos. Sin embargo, es importante desarrollar investigaciones alrededor de cómo confluyen los diferentes tipos de conocimiento necesarios en la constitución de una idea de diseño a partir de los niveles de desarrollo planteados por los estudiantes, pues si bien la autora sigue los planteamientos de otros autores al considerar un proceso de modelación donde los conocimientos de las áreas implicadas no se subordinen entre sí, este proceso resulta complejo porque diferentes situaciones pueden requerir un mayor nivel de conocimiento de otras áreas.

\section{Aportes y discusión}

Este trabajo involucró dos ambientes de modelación. El primero se centró en la inmersión de tareas de modelación, a partir de las cuales se logró dinamizar el conocimiento matemático e ingenieril, al promover articulaciones entre ellos frente a los conceptos de área, volumen y funciones (por mencionar algunos ejemplos). Los resultados en este primer ambiente muestran que los futuros ingenieros logran relacionar sus vivencias con su futuro desarrollo profesional; además, reflexionan, analizan y toman decisiones a la luz de una idea de diseño considerando los conceptos matemáticos involucrados. El segundo ambiente de modelación consistió en un proceso de indagación que los estudiantes realizaron, según sus intereses, sobre un proyecto de modelación; en este contexto se posibilitó la reflexión sobre qué se modela, quién modela, cómo modela, y cuáles son las condiciones que deben garantizarse para modelar en coherencia con las demandas de un usuario y del producto en general, para finalmente dar solución a un problema determinado.
En una discusión en clase sobre qué es lo que se modela y qué condiciones se debían considerar al enfrentarse a una tarea en la cual debían analizar los costos de producción de un empaque con cartón industrial, uno de los estudiantes reconoció, frente a la pregunta ¿Cuántos empaques pueden realizarse por pliego?, que los valores que ofrecía la calculadora al realizar una división entre áreas eran diferentes a los que debía encontrar en la realidad, pues el área que se obtiene a través de la división no necesariamente se puede ubicar en el pliego. Esta situación da cuenta del sentido que debe cobrar la matemática en el contexto ingenieril porque más allá de las medidas realizadas y los procedimientos efectuados se encuentra la interpretación de lo que ello significa y lo que implica para realizar el diseño requerido bajo las condiciones económicas consideradas como las de estructura de los empaques. También es importante considerar que los conceptos matemáticos en este caso emergen del contexto ingenieril a la hora de determinar las condiciones para resolver al 
problema de diseño, más que llevar conceptos predeterminados a una situación en la cual tenga sentido su aplicación.

Dentro de los resultados se muestran evidencias que dan cuenta que las discusiones en clase posibilitaron el reconocimiento de variables y factores que los estudiantes deben tener en cuenta a la hora de diseñar un producto. En este sentido, la interacción entre estudiantes y maestros aportó a la resolución de las tareas de modelación, pero también contribuyó a generar nuevos cuestionamientos que posibilitaron a los estudiantes considerar otros elementos y variables. Estos aspectos son importantes porque revelan los aportes de las discusiones, comentarios y valoraciones de los demás a la hora de plantear la solución a un problema.

Otro de los aportes de este trabajo se centra en la concepción de modelación matemática situada y sobre las condiciones para lograr articulaciones entre el campo de acción de una clase particular de ingenieros y la matemática. En coherencia con ello, en este estudio la modelación matemática bajo este enfoque posibilitó la apropiación conceptual tanto de la matemática como de la ingeniería y el diseño de producto, aportó a definir los requerimientos formales y estructurales que deben considerarse a la hora de diseñar un producto bajo las condiciones de un usuario y mediante el uso de recursos tecnológicos, estrategias de solución, dialogo con expertos, entre otros.

Pese a lo anterior, este trabajo de investigación suscita algunos cuestionamientos relacionados con las posibilidades de incorporar la modelación matemática en otros contextos y áreas del conocimiento; por ejemplo, ¿qué características deben considerar los ambientes de modelación matemática para promover articulaciones entre la mate- mática y otras ingenierías? ¿Qué consideraciones deben tenerse en cuenta para incorporar una modelación matemática situada en otros campos de estudio? Esto es importante porque saber matemática no implica saber usarla en otros contextos. Además, es necesario que haya relación entre las actividades planteadas y las necesidades de formación en el campo específico de formación.

Bajo los planteamientos anteriores, la pregunta sobre los diferentes roles que puede tener la modelación matemática desde una perspectiva situada sigue siendo relevante tanto en la formación ingenieril como en educación matemática. Esta investigación plantea, al menos, dos aspectos de interés; el primero es que la modelación matemática situada debe considerar el campo de formación de los estudiantes para posibilitar ambientes que contribuyan a su desarrollo profesional; el segundo es que el proceso de modelación matemática bajo esta perspectiva devela unas condiciones particulares, propias del contexto, que no están en correspondencia con los ciclos de modelación matemática planteados por algunos investigadores. Esta última discusión también ha sido planteada por autores como Albarracín, Arleback, Civil y Gorgorió (2019) quienes manifiestan que los diagramas de modelación no parecen revelar toda la complejidad de algunos procesos para resolver problemas (otros aportes de interés se pueden encontrar en Rendón-Mesa, Duarte, Villa-Ochoa, 2016), a partir de estas contribuciones se contempla la posibilidad de extender y reorganizar los ciclos de modelación, acorde a las prácticas situadas y necesidades de estos futuros profesionales.

Si bien la inmersión de ambientes de modelación matemática bajo esta perspectiva en la formación de ingenieros es importante bajo las consideraciones anteriores, 
esta implica establecer relaciones con otras disciplinas y expertos, para lo cual se debe tener en cuenta el tiempo requerido para ello, haciendo que se torne complejo el cumplimiento con los propósitos de aprendizaje. Adicionalmente, se debe reflexionar sobre las posibilidades de establecer diálogos con colegas en la formación de ingenieros y las transformaciones curriculares que implican ciertas estrategias como la resolución de problemas y que son importantes para atender a las necesidades de formación de los estudiantes.

\section{REFERENCIAS BIBLIOGRÁFICAS}

Albarracín, L., Arleback, J., Civil, E., \& Gorgorió, N. (2019). Extending Modelling Activity Diagrams as a tool to characterise mathematical modelling processes. The Mathematics Enthusiast, 16(1), 211-230.

Blomhøj, M. (2004). Mathematical modelling: a theory for practice. En B. Clarke, D. M. Clarke, G. Emanuelsson, B. Johansson, D. V. Lester, A. Wallby, \& K. Wallby (Eds.), International Perspectives on learning and teaching mathematics (pp. 145-159). Göteborg University: National Center for Mathematics Education.

Blum, W. (2011). Can modelling be taught and learnt? Some answers from empirical research. En Trends in teaching and learning of mathematical modelling (pp. 15-30). Springer, Dordrecht.

Bravo-Bohórquez, A., Castañeda-Rodriguez, L. J., Hernández-Yomayusa, H. I., \& Hernández-Hernández, L. A. (2016). Enseñanza de las matemáticas en ingeniería: Modelación matemática y matemática contextual. Revista Educación en Ingeniería, 11(21), 27-31.

Plaza, L.F. (2016). Obstáculos presentes en modelación matemática. Caso ecuaciones diferenciales en la formación de ingenieros. Revista Científica, 25, 176-187. Doi: 10.14483/udistrital.jour. RC.2016.25.a1

Rendón-Mesa, P. A., Duarte, P. V. E., y Villa-Ochoa, J. A. (2016). Articulación entre la matemática y el campo de acción de la ingeniería de diseño de producto: componentes de un proceso de modelación matemática. Revista de la Facultad de Ingenieria U.C.V., 31(2), 21-36.

Rendón-Mesa (2016). Articulación entre la matemática y el campo de acción de la ingeniería de diseño de producto: aportes de la modelación matemática. Doctorado en Educación, Facultad de Educación, Medellín: Universidad de Antioquia.

Villa-Ochoa, J.A., \& Berrío, M. J. (2015). Mathematical Modelling and Culture: An Empirical Study. En G. A. Stillman, W. Blum, \& M. S. Biembengut (Eds.), Mathematical Modelling in Education Research and Practice, International Perspectives on the Teaching and Learning (pp. 241-250). Cham: Springer. https://doi.org/10.1007/978-3-319-18272-8_19

Villa-Ochoa, J., Castrillón-Yepes, A., y Sánchez-Cardona, J. (2017). Tipos de tareas de modelación para la clase de matemáticas. Espaço Plural, 18(36), 219-251. 